\title{
El aporte de la epistemología del derecho económico en la formación de abogados
}

\section{The contribution of the epistemology of economic law in the training of lawyers}

\section{Carlos F. Forero Hernández ${ }^{1}$}

Así como el 'huevo y la gallina', el derecho y la economía no acaban de situarse en un orden de prelación que resulta innecesario, pues ambas formas de organización son pilares en cualquier estructura social. Las nuevas vertientes económicas, en especial el neoliberalismo, han determinado el tipo de Estado y control legal de la forma de manifestación de estas vertientes; hoy por hoy, los avances cientificos y tecnológicos han revolucionado las economías mundiales $y$, lamentablemente, lo que va a la postre es el derecho (Gómez Gallardo, 2006, p. 53).

O

Recepción: 25/01/2021
0

Aprobación: 26/11/2021
O

Publicación: 22/12/2021

Para citar este artículo

Forero Hernández, C. F. (2021). El aporte de la epistemología del derecho económico en la formación de abogados. Indagare, (9), 46-61. https://doi.org/10.35707/indagare/905

1 Coordinador del Semillero de Investigación de Derecho Administrativo Jaime Vidal Perdomo, Universidad de Ibagué, Ibagué, Colombia. ORCID: 0000-0002-9108-2413.

Correo electrónico: carlos.hernandez@unibague.edu.co 


\title{
Resumen
}

El escrito pretende hacer una reflexión acerca de la relación economía y derecho, así como de la noción del derecho económico. Se expondrá, además, sobre el aporte de la epistemología del derecho económico en la formación de abogados. El texto corresponde a una de las actividades académicas del semillero de investigación de Derecho Administrativo Jaime Vidal Perdomo, al revisar temas como huida del derecho administrativo hacia el derecho privado, derecho administrativo económico e intervención del Estado en la economía.

\begin{abstract}
The paper intends to reflect on the relationship between economics and law, as well as on the notion of economic law. It will also address the contribution of the epistemology of economic law in the training of lawyers. The text corresponds to one of the academic activities of the research group of Administrative Law Jaime Vidal Perdomo, reviewing topics such as the flight of administrative law towards private law, economic administrative law and State intervention in the economy.
\end{abstract}

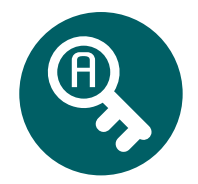

Palabras claves: Economía y derecho, derecho económico, intervención del Estado en la economía.

Key words: Economics and law, economic law, State intervention in the economy.

\section{Introducción}

En este documento se pretende realizar una reflexión sobre la discutida relación economía y derecho, así como de la noción del derecho económico. Se plasman, además, dos conclusiones principales: la primera, el estudio de la discutida relación economía y derecho ya se encuentra superado y, por tanto, se debe dar paso al estudio del derecho económico; la segunda, se recomienda que en todos los cursos del programa de Derecho de la Universidad de Ibagué analicen las funciones económicas de cada una de las instituciones jurídicas, en aras de obtener una visión más amplia sobre ellas, como uno de los aportes del estudio de la epistemología del derecho económico en la formación de abogados.

Debe precisarse que el texto fija nociones muy elementales de los temas, dado que detenernos en ellas significará todo el estudio de la asignatura Economía y derecho, 
aunque tiene muy mal puesto su nombre, y para ello se hace por medio de un libro, no en pocas páginas. El autor consigna su especial gratitud a la integrante del semillero de investigación de Derecho Administrativo Jaime Vidal Perdomo, Paula Yulieth Arana Guaraca, quien colaboró, respecto de la recolección de información para la construcción del documento, así como también, lo motivó a cumplir este objetivo.

\section{Derecho económico e intervención del Estado en la economía}

\subsection{Noción de economía y sus escuelas de pensamiento económico}

De la economía se han ofrecido tantas nociones como autores se han ocupado del tema. La más compartida por los economistas (eso sí en forma de parafraseo) es la siguiente: aquella área de conocimiento ${ }^{2}$ que analiza la producción, distribución y consumo tanto de bienes como de servicios o de recursos escasos en una sociedad. Es de precisar que en todas las nociones resultantes de los estudiosos asocian la característica de escasez y aluden a situación de dificultad frente al logro de satisfacción de todas las necesidades humanas ${ }^{3}$.

Algunos expertos advierten que el objeto de estudio de la economía radica en el análisis de fenómenos sociales que ocurren en los procesos o actividades que involucran situaciones de escasez y de satisfacción de necesidades. Palacios Mejía (1999), por ejemplo, ha aclarado que la economía "se ocupa solo de aquellos bienes y servicios que son escasos en relación con lo que los miembros de la sociedad podrían desear. Sin escasez no existirían problemas económicos" (p. 4). Por tanto, la economía debe ser estudiada siempre con esta institución.

No podemos perder de vista que el origen de la economía (o de la ciencia económica, para algunos), dado que se encuentra en las más profundas raíces de la filosofía, de la política y de la ética; más en las de la filosofía política en la medida en que esta proporcionaba (y todavía proporciona), no solo a economistas sino también a otros profesionales, elementos de juicio valiosos para ser buenos interlocutores en los discursos tanto académicos como profesionales.

Para efectos de este trabajo se expone la noción ofrecida por Chang (2014) quien entiende por economía que "es el estudio de la actividad económica" (p. 29). Se debe advertir que lo anterior es realizado tanto por los particulares como por el Estado (contratación estatal, por ejemplo); es decir, se frecuenta en el derecho privado y público. Ahora, en caso de distorsiones o irregularidades (estudiados también como fallos) que se presenten en dichas actividades surge la necesidad de intervención por parte del Estado; esto equivale a derecho económico y es entendido como el derecho de la dirección e intervención del Estado en la economía (Forero Hernández, 2019).

\footnotetext{
2 Se resalta que algunos consideran que la economía es una ciencia mientras que otros advierten que no lo es.

3 Desde luego se han encontrado textos precisando que la escasez se trata de situación de imposibilidad y no de dificultad respecto del logro de satisfacción de todas las necesidades humanas.
} 
Se resalta que la economía se clasifica en positiva (ser) y normativa (deber ser). La primera se divide en economía descriptiva (descripción de la realidad económica, así como el comportamiento de la sociedad) y teoría económica (formulación de teorías, leyes o modelos, con base en la economía descriptiva). La segunda, estudia la política económica ${ }^{4}$ en ella se considera la política monetaria (su finalidad es controlar la inflación), política fiscal (analiza la cobertura del gasto público con los ingresos recaudados; y tiene estrecha relación con el control de la tasa de desempleo) y política cambiaria (su propósito es controlar la tasa de cambio en una economía).

También se estudia con frecuencia, y de manera paralela con las escuelas de pensamiento económico (que más adelante serán mencionadas y explicadas, eso sí, no a profundidad), las instituciones de microeconomía y macroeconomía. La primera se identifica con el estudio de la economía en relación con acciones individuales (consumidores, por ejemplo). La segunda, con el estudio de magnitudes colectivas o globales (exportaciones, si es el caso). Se debe reconocer que sobre estos temas se han escrito suficientes reflexiones y apreciaciones.

Estas instituciones (microeconomía y macroeconomía) asocian para su estudio interesantes temas como la oferta (conjunto de bienes y servicios puestos en el mercado) y la demanda (conjunto de bienes y servicios adquiridos o realizados), el desempleo (está en condiciones de trabajar, pero no tiene empleo o lo ha perdido), la inflación (elevación del nivel general de precios de bienes y servicios en una economía), políticas económicas (herramientas económicas de intervención estatal para corregir las distorsiones del mercado o de la economía), producto interno bruto (PIB) (medida de valor de la actividad económica - producción de bienes y servicios- de un país), etc.

En la literatura, por su parte, se exponen muchas escuelas económicas con grandes e importantes aportes orientadas a la solución de distintos y complejos problemas socioeconómicos. En este apartado se relacionarán las principales (Chang, 2014). La escuela clásica profundiza en la producción como el ámbito más importante de la economía, es de la idea según la cual la economía cambia por la acumulación de capital (la inversión). Esta es partidaria del libre mercado.

La escuela neoclásica nos hace reflexionar que son el comercio y el consumo los ámbitos más importantes de la economía y, para ella, estos se asocian a las elecciones individuales; además, apoya tanto el libre mercado como del intervencionismo del Estado ante la existencia de fallos del mercado o de la economía. El énfasis de estudio de la escuela marxista es la producción, y la economía cambia por la lucha de clases, la acumulación de capital; así como el progreso tecnológico; esta escuela, por su parte, recomienda como política económica aplicar la planificación central.

4 No se debe confundir con la economía política y esta consiste en una de las aplicaciones de la política económica. 
La escuela desarrollista (aunque para algunos no es una escuela) también estudia la producción como ámbito más importante de la economía y profundiza en el desarrollo de las capacidades productivas; es además partidaria de la idea de protección e intervención del Estado ante los fallos del mercado. El eje central de la escuela austríaca es el comercio, así como a las elecciones individuales en la adquisición de bienes y servicios; es seguidora del libre mercado.

La escuela schumpeteriana profundiza en la producción y recomienda emplear la innovación tecnológica como aplicación de globalización (o economía sin fronteras) digitalizada. La escuela keynesiana de manera tímida ${ }^{5}$ estudia la producción; sus mayores énfasis de estudio son las políticas fiscales y la redistribución de la renta en favor de los más pobres; ahonda también en la política tributaria y el gasto público. Esta escuela es partidaria de la teoría de la dirección e intervención del Estado en la economía.

La escuela institucionalista también de manera tímida aborda la producción y para ella la interacción entre las personas y las instituciones (formales e informales) son sus mayores ejes de análisis; es partidaria de la aplicación del estudio de los costes de transacción. La escuela conductista estudia la producción y asocia las conductas, así como las elecciones racionales; acepta que el Estado intervenga ante los fallos del mercado.

En nuestro sentir, en todos los aportes y explicaciones ofrecidas por estas escuelas, de alguna manera se estudian la teoría de la dirección e intervención del Estado en la economía (y esto equivale a derecho económico). Se afirma así por cuanto la presencia del Estado es indispensable para corregir las disfuncionalidades del mercado; de ahí que el Estado intervendrá para racionalizar la economía en aras de obtener el mejoramiento de la calidad de vida de los habitantes, la distribución equitativa de las oportunidades y los beneficios del desarrollo, así como la preservación de un ambiente sano.

Ante la existencia de varios aportes y explicaciones de cada una de las escuelas nos permiten afirmar que la economía no es un área de conocimiento exacta. Se asegura de esa manera por cuanto también está mediada por las vicisitudes y ambigüedades del lenguaje; además, el mundo fáctico (de los hechos) es bastante complejo, dinámico y está sujeto a cambios más confusos y acelerados. No menos importante es destacar que algunos consideran que la economía es un área de conocimiento exacta a partir del buen uso de análisis e interpretación de fenómenos sociales, así como también por tratarse de una ciencia (social).

Debemos reconocer que la aplicación de la teoría de la intervención del Estado en la economía ha tenido tanto defensores como detractores. Tal como lo explica

Decimos tímida porque en algunas ocasiones la comparte y en otras no. 
Varó (2013), los que están en contra argumentaron (y siguen advirtiendo) que las intervenciones estatales en los procesos económicos son las que provocan las crisis, las que alteran el libre mercado, convirtiendo espacios planificados y generando profundos desequilibrios. Discusión que se encuentra vigente. En todo caso, gústenos o no, el Estado siempre estará presente en la vida económica y la mayoría de las constituciones (la nuestra, por ejemplo) le han asignado la tarea de dirección e intervención en la economía en caso de disfuncionalidades.

Claro está que las intervenciones por realizar deben ser prudentes, justificables, razonables, proporcionales y acordes con los postulados de la fórmula Estado social de derecho. Por eso tienen razón Kresalja Roselló y Ochoa Cardich (2017) al advertir que toda intervención "debe estar limitada en la constitución como garantía mínima para los ciudadanos y los agentes económicos, dado que la inclusión frondosa e indiscriminada de cláusulas constitucionales económicas puede dar lugar a graves distorsiones" (p. 22).

Podríamos afirmar que no resulta desacertado al referir que la dirección e intervención del Estado en la economía es como el aire por cuanto está en todas partes. Tal explicación a simple vista es comprensible, desde luego se admite cualquier crítica, pues de eso se trata. En este sentido, resulta pertinente citar la explicación ofrecida por Vidal Perdomo (1966) al establecer que la noción de intervención del Estado (desde una óptica amplia) siempre ha existido:

La acción administrativa procede o es consecuencia de la intervención del Estado. La noción elemental de intervención se refiere a las relaciones entre gobernantes y gobernados, a la cantidad de órdenes y prescripciones que pueden dar los primeros y que sean norma de conducta de los segundos. Entendida así la intervención del Estado, forzoso es concluir que siempre ha existido, pues este fue siempre depositario de una autoridad que se impone a los ciudadanos, así como el gestor de una serie de servicios necesarios a la vida en común y que no podía ser atendido por los particulares. Sin embargo, lo que se conoce con el nombre de intervencionismo estatal no es el fenómeno en sí, sino su aumento extraordinario del presente siglo, principalmente, producto de nuevas concepciones sobre el Estado y su papel en la vida de los pueblos. (p. 158).

Se tiene entonces que en el estudio de la economía debe abordarse estas cuatro áreas principales: a) la historia del pensamiento económico (de las escuelas económicas); b) los métodos de análisis económicos (que son los aportes de cada una de las escuelas); c) la microeconomía y d) la macroeconomía. Se repite, todas ellas estudian de alguna manera la teoría de la dirección e intervención del Estado en la economía (podríamos decir que no son cuatro sino cinco áreas), como una de las aplicaciones de la relación economía y derecho. 
En relación con lo anterior, sin pretender que el economista o el politólogo, por ejemplo, sea un filósofo, estas áreas resultan importantes para su análisis en la medida en que aportan elementos de juicio valiosos para ser buenos interlocutores en las disertaciones tanto académicas como profesionales; en aras de ser capaces de intervenir interdisciplinariamente (Forero Hernández \& Arana Guaraca, 2020).

No menos importante es señalar que, de acuerdo con la doctrina del derecho penal económico, existen ilícitos que hacen que dificulten (o impiden, para algunos) la labor de dirección e intervención del Estado en la economía, como son, entre otros, los delitos económicos. Ante la existencia de esos ilícitos contra la economía, el Estado debe actuar estableciendo reglas de juego y es así como gestan el derecho penal económico, el derecho administrativo sancionatorio (en él se encuentra, por ejemplo, el derecho administrativo sancionador tributario), entre otras instituciones jurídicas. Todo eso sin duda alude a una de las aplicaciones de la relación economía y derecho. En las siguientes páginas se explicará dicha relación ${ }^{6}$, que es una de las grandes temáticas del derecho económico y surge, por su estudio en conjunto entre estas dos áreas de conocimiento, el derecho económico.

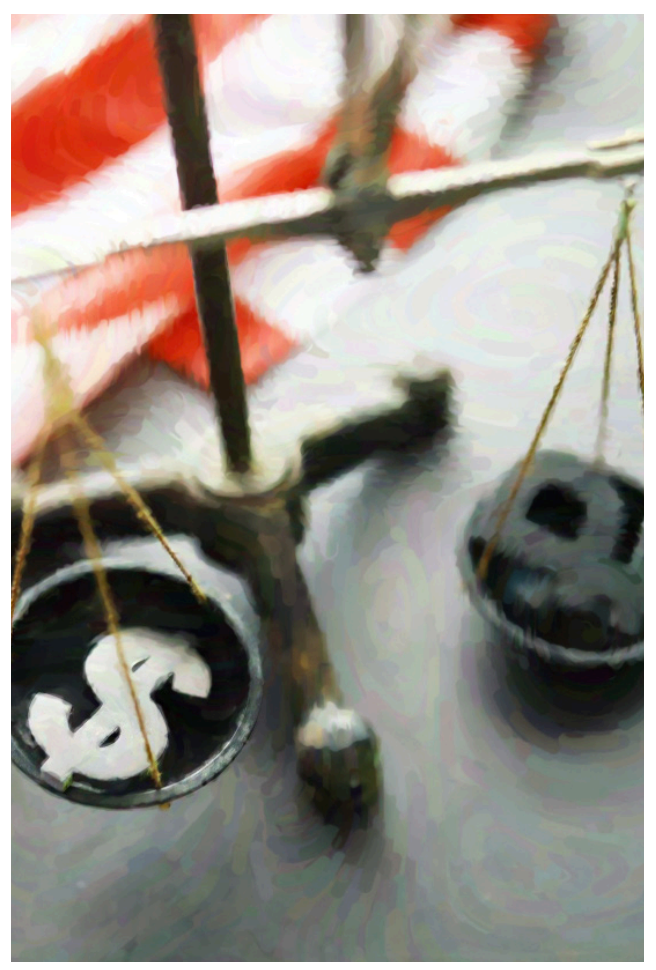

2.2. El estudio en conjunto entre economía y derecho gesta el derecho económico

En principio, en la academia, así como en las escuelas de pensamiento económico con la participación de los clásicos Weber y Smith, se había discutido mucho acerca de la existencia de la relación entre economía y derecho (Franco Vargas, 2013). En la actualidad, esta discusión se encuentra resuelta, superada, hasta el punto en que muchos han difundido con frecuencia la denominación del derecho económico que estudia en conjunto estas dos áreas de conocimiento. Para Franco Vargas (2013), en razón a la similitud entre sí, a partir del análisis de la conducta del individuo (como objeto de estudio) surge el derecho económico. Igualmente se ha profundizado como otro campo de

6 Algunos establecen "relación entre la economía y el derecho". No la compartimos por cuanto las expresiones la y el indican que los temas se encuentran agotados y no lo están. Hay mucho por debatir. 
aplicación de dicha relación: el Análisis Económico del Derecho (AED), como aquel instrumento que examina las normas jurídicas con criterios económicos y es muy importante en la escuela neoclásica.

Algunos han considerado sin tanto rodeo que el derecho económico y el AED son sinónimos; son lo mismo. Esta explicación no está exenta de críticas. Desde nuestra perspectiva, el derecho económico es un área de conocimiento (o una rama del derecho, si se prefiere) en tanto que el AED es una herramienta económica para analizar normas jurídicas. Gutiérrez Prieto (2001), por su parte, presenta otro argumento muy interesante acerca de la diferencia entre el AED y el derecho económico:

A pesar de la similitud en los términos, Law and Economics no puede ser identificado con el derecho económico. Se trata de dos formas de conocimiento que disponen de diferencias tanto en razón de sus objetos como en razón de sus metodologías. En efecto, mientras que Law and Economics es, fundamentalmente, análisis económico (en otros términos, mientras que Law and Economics es economía), el derecho económico es derecho. Más aún, Law and Economics no puede ser entendido ni como una versión del derecho económico ni, tampoco, como una metodología propia, mucho menos exclusiva, de esta disciplinar jurídica. (p. 53).

Se enuncia la advertencia otorgada por Posner citado por Amador Cabra (2014) cuando señala que el AED no posee la pretensión de reemplazar la lógica jurídica sino complementarla a través del uso de la economía en relación con los fenómenos legales. Para Amador Cabra (2014), el AED al abordar el derecho desde una perspectiva económica implica el desarrollo de estos tres elementos: a) la interpretación y análisis de las normas jurídicas se realiza a partir de una óptica económica; b) la racionalidad con que se dota al sistema normativo es la misma que usa la teoría microeconómica en su construcción teórica; c) el entorno legal produce incentivos para que los agentes se comporten de manera eficiente.

No sobra indicar que ante la dificultad de ofrecer un sinnúmero de respuestas a cargo de la teoría neoclásica frente a todos y cada uno de los complejos problemas socioeconómicos surgió la Nueva Economía Institucional (NEI), que pertenece a la escuela institucionalista y, para ella, las instituciones (formales e informales) económicas, sociales y políticas son la base del crecimiento económico (en muchas ocasiones asocia el estudio del crecimiento con la relación economía y desarrollo).

Por su parte, uno de los debates más fuertes que hemos identificado es si el derecho depende de la economía o si esta última depende de él. Creemos que se trata de una discusión interminable (intemporal). En todo caso resulta conveniente estudiar en conjunto en aras de aportar soluciones económicas y jurídicas frente a los distintos y complejos problemas sociales. Es más, se ha demostrado que los problemas sociales 
se resuelven no solo desde la economía sino con el concurso de otras disciplinas, con el derecho, por ejemplo. A decir verdad, la economía necesita de un marco jurídico para poder desenvolverse. De ahí que del análisis en agrupación entre estas dos áreas de conocimiento surge el derecho económico.

\section{3. Nociones de derecho económico e intervención del Estado en la economía} Como del estudio en conjunto entre economía y derecho gesta el derecho económico, surge entonces la necesidad de averiguar acerca de su noción, aunque se debe reconocer que no hay una única o universal. Resulta indispensable definir primero las instituciones de economía y derecho. Por economía entendemos como aquella área de conocimiento que estudia la actividad económica, que es el postulado ofrecido por Chang (2014). Se resalta que dicha actividad es objeto de intervención del Estado en caso de irregularidades o distorsiones.

Por otro lado, por derecho entendemos como aquel conjunto de reglas dictadas y promulgadas por los órganos competentes orientadas a regular (o intervenir) las relaciones sociales y económicas ${ }^{7}$. El Estado, a través de sus autoridades competentes, regula las relaciones jurídicas (privadas o públicas). Se debe registrar que no hay una idea única o universal acerca del derecho. Hugo Guerriero (2007), por ejemplo, ha reconocido que elaborar una noción del derecho no resulta tarea fácil y considera, además, que "el derecho no es un conjunto de normas reguladoras de la conducta por cuanto él simplemente la califica. Las normas contienen el derecho y en tal caso son jurídicas; de lo contrario, serán imposiciones" (p. 99).

Si la economía tiene sus escuelas de pensamiento, el derecho también posee sus seminarios (por así decirlo) y con grandes aportes para la solución de problemas sociales. Veamos las principales, eso sí, no a profundidad (Alfonso Vargas, 2020). La escuela iusnaturalista hace mayor énfasis en la relación entre derecho y moral; se analiza con reiteración el derecho natural. Para ella, el derecho natural alude a que es Dios quien determina aquello que es justo y bueno y cuyas normas se fundamentan en los principios morales y estos últimos son vistos como axiomas imperativos. Esta escuela reivindica que una norma que no se fundamente en principios morales no puede ser catalogada como jurídica y mucho menos como válida.

La escuela positivista estudia con frecuencia la división tajante entre derecho y moral; para ella no es de recibo la moral y analiza con frecuencia el derecho (positivo) vigente. También se analiza el derecho creado y aplicado por los hombres. Esta escuela no desconoce toda fundamentación de derecho natural, pero sí se aparta de las afirmaciones religiosas. Para el derecho natural se reivindica del valor de orden de

Se resalta que algunos estudiosos consideran que el derecho es una ciencia mientras que otros afirman que no lo es. 
aquello que es justo, para el derecho positivo es justo lo que es ordenado y por el hecho de ser ordenado.

La escuela del realismo jurídico resalta que el derecho debe ser abordado en conjunto con los fenómenos sociales, con las transformaciones sociales. Critica el derecho estático. Para ella, el derecho dinámico es de recibo porque es ajustado a la realidad. Es partidaria de la eficacia normativa, esto es, del cumplimiento de una norma positiva según las exigencias de las transformaciones sociales.

La escuela no positivista reivindica que no todo se resuelve con normas jurídicas, sino también con principios, dado que son vistos como axiomas imperativos y cuya inobservancia se hará acreedor de sanción. Difunde con frecuencia la aplicación del principio de proporcionalidad o de razonabilidad a los casos difíciles; este axioma posee tres estándares: necesidad, idoneidad y proporcionalidad en sentido estricto ${ }^{8}$.

Ante la existencia de varios aportes y explicaciones de cada una de las escuelas nos permiten concluir que el derecho no es un área de conocimiento exacta. En verdad es una ciencia inexacta. Se afirma de esa manera por cuanto el derecho igualmente está mediado por las vicisitudes y ambigüedades del lenguaje; además, el mundo fáctico (de los hechos) es muy complejo, dinámico y está sujeto a cambios más confusos y acelerados que el derecho.

Conviene manifestar que en la literatura se ha identificado que, frente a determinados casos, difíciles en algunas ocasiones, se utilizan el principio de proporcionalidad y en otras el AED y, por tanto, determinados juristas han discutido acerca de cuál es el instrumento (más) idóneo para facilitar soluciones a los problemas sociales. En nuestro sentir, frente a los problemas sociales no hacen daño si utilizamos en conjunto estas dos herramientas hermenéuticas por cuanto contribuyen a aportar varias soluciones, aunque no sean las (más) óptimas, pero sí legítimas (justificables). Recordemos, además, que el AED no pretende reemplazar la lógica jurídica sino complementarla a través de la ciencia económica en relación con los fenómenos legales.

Por eso el estudio y el empleo de estas herramientas hermenéuticas resultan trascendentales para el abogado, así como para quienes están en formación, en la medida en que proporcionan elementos de juicio valiosos para ser buenos interlocutores en los discursos tanto académicos como profesionales; en aras de convertirse en personas capaces de intervenir de forma interdisciplinaria (Forero Hernández \& Arana Guaraca, 2020). Son las bases necesarias para formar un abogado integral para el mundo sin fronteras.

Por su parte, la más importante división de las ramas del derecho es aquella que la clasifica en normas de derecho público (sus ramas son, entre otras, el derecho penal, administrativo, constitucional, procesal, internacional público, tributario) y de

8 Resulta pertinente señalar que la jurisprudencia de la Corte Constitucional no ha realizado la distinción del principio de proporcionalidad con el de razonabilidad; utiliza de manera indiscriminada estas denominaciones 
derecho privado (sus ramas son, entre otras, el derecho civil, comercial, internacional privado, laboral). Algunos estudiosos, entre ellos Barón Barrera (2016), consideran la conveniencia de hablar de la existencia de un derecho mixto y este se identifica con el derecho económico. Así, para Barón Barrera (2016), el derecho económico es un derecho mixto en relación con el público y el privado. Se debe reconocer, además, que todas las ramas del derecho están involucradas con elementos económicos; de eso no existe la menor duda.

Ahora bien, del derecho económico se han brindado tantas nociones como autores se han ocupado del tema. Creemos que no existe un criterio clave absoluto para definir el derecho económico y se afirma así, por cuanto se está frente a un derecho heterogéneo y dinámico. Es más, este derecho depende en gran parte del papel del Estado. Es decir, está condicionado por su concreta organización y funcional. De hecho, el Estado como fenómeno histórico asociado al desarrollo (ora social, político, económico y cultural) siempre ha tenido una función importante con base en la economía, dirigida e intervenida en cada periodo. De esta manera, si la organización y funcional del Estado cambia, el derecho económico también debe hacerlo. Así que elaborar una noción exacta acerca del derecho económico no es tarea fácil.

Algunos refieren que el derecho económico es un derecho híbrido en concordancia con el derecho público y el derecho privado (Forero Hernández, 2019). Para otros, es el derecho del desarrollo económico, que asocia para su estudio temas de desarrollo y sostenibilidad. Un buen número de estudiosos afirma que es aquel derecho de la regulación económica que es la misma explicación según la cual el derecho económico es el derecho de la ordenación económica.

Barón Barrera (2016) considera que ante el fenómeno de la huida del derecho administrativo que se identifica con la aplicación con mayor rigor o intensidad de normas de derecho privado (normas comerciales y civiles), en algunos escenarios de la administración pública (contratación estatal, por ejemplo), el derecho administrativo (como aquel derecho de la administración pública) se está trasformando en derecho económico. Frente a este fenómeno, Ariño Ortíz (2013) ha indicado que existen tres vías fundamentales en las que se ha manifestado la tendencia de dicha huida: a) mediante la constitución de empresas públicas; b) a partir de la utilización de formas, categorías y régimen jurídico privado para la realización de actividades de orden administrativo; c) a través de la privatización del propio derecho administrativo público.

Para efectos de este texto, lo entendemos como el derecho de la dirección e intervención del Estado en la economía, como aquel conjunto de normas jurídicas que facilita las intervenciones del Estado en el aspecto específico de la producción, 
distribución y consumo de bienes y servicios. Como la economía estudia la institución de actividad económica, esta última es objeto de intervención del Estado ante la existencia de disfuncionalidades y eso equivale a derecho económico. Todas estas explicaciones son nociones keynesianas.

Por sus distorsiones o disfuncionalidades, la economía requiere de la intervención del Estado. Así, su presencia es indispensable para corregir las disfuncionalidades del mercado; intervendrá para racionalizar la economía con el fin de conseguir el mejoramiento de la calidad de vida de los habitantes, la (justa) distribución equitativa de las oportunidades y los beneficios del desarrollo, así como lo más importante: la preservación de un ambiente sano.

La presencia del Estado en la economía es trascendental para corregir las distorsiones o fallos del mercado y esto equivale a derecho económico. A su turno, tal intervención está precedida y acompañada de una constante vigilancia y control de la economía, pues recordemos que el mercado es incapaz de darse sus propias reglas. La economía no puede existir por sí misma sin la existencia y la acción previa del Estado. De eso no existe la menor duda. Tal dirección e intervención constituye entonces una tarea prioritaria en el contexto de un Estado social de derecho.

Por eso se exponen las reflexiones de Chang (2014) al enunciar que la categoría de dirección e intervención del Estado en la economía es el mejor invento de la humanidad por cuanto está ideada para corregir las disfuncionalidades del mercado o de la economía: "Es innegable que el Estado sigue siendo la tecnología organizativa más poderosa que ha inventado la humanidad, por lo que es muy difícil alcanzar grandes cambios económicos (y sociales) sin su ayuda” (p. 362).

En Colombia, esta institución de intervención cuenta con fundamento constitucional a partir del artículo 334 de la Constitución Política. Por tanto, se encuentra constitucionalizada. Resulta pertinente citar la noción ofrecida por Hernández Quintero (1991) respecto de esta institución, dado que es quien señala que es "el conjunto de normas con las cuales se pretende regular y delimitar la autonomía de los particulares a fin de garantizar el logro de los intereses de la comunidad, esto es, el bien común" (p. 21). Esta es una figura o categoría jurídica que contribuye al cumplimiento adecuado de los fines esenciales del Estado.

En relación con lo anterior, hemos considerado que la aplicación de la dirección e intervención del Estado en la economía contribuye a materializar los postulados de la fórmula Estado social de derecho (Constitución Política, 1991, artículo 1) y así lo da a entender la Corte Constitucional en la Sentencia C-150 de 2003 cuando plantea que esta (la intervención estatal en la economía) "debe garantizar la efectividad de los principios del Estado social de derecho" (p. 24). En sentido coincidente lo explica Franco Vargas (2013) al indicar lo siguiente: 
El Estado social de derecho define el particular diseño institucional dirigido a favorecer la intervención del Estado en la economía y ello se manifiesta en la gestión directa por parte de los entes públicos de algunas actividades especiales como la prestación de servicios públicos, las políticas de redistribución de la riqueza, el fomento de la industria y del empleo y, en general, la preocupación por garantizar el bienestar de la sociedad. (p. 26).

En este orden de ideas, es fácil afirmar que el sistema económico en Colombia es un sistema híbrido - derechos y límites a la vez- por cuanto la Constitución Política posibilita a los particulares llevar a cabo actividades económicas (libertad de empresa), pero con un gran e importante límite constitucional consistente en la susceptibilidad de intervención del Estado en caso de disfuncionalidades o distorsiones de las mismas. Lo anterior se identifica, de acuerdo con la doctrina constitucional, con el sistema de economía social de mercado (Sentencia C-263, 2011). Sobre el sistema económico adoptado en Colombia en vigencia de la fórmula de Estado social de derecho, resulta pertinente citar la explicación dada por la Corte Constitucional:

La Constitución de 1991 al adoptar un modelo de Estado social de derecho, introdujo un modelo de economía social de mercado en el que, de un lado, se admite que la empresa es motor de desarrollo social y por esta vía se reconoce la importancia de una economía de mercado y la promoción de la actividad empresarial, pero por otro, se asigna al Estado no solo la facultad sino la obligación de intervenir en la economía con el fin de remediar las fallas del mercado y promover el desarrollo económico y social. (Sentencia C-263, 2011, p. 9).

Para Franco Vargas (2013), el sistema económico adoptado en Colombia es de economía mixto porque:

Si bien la norma fundamental del sistema jurídico reconoce que muchas de las actividades económicas pueden ser realizadas por los particulares de manera descentralizada dentro del mercado y que se reconoce el derecho a la propiedad privada (lo cual configura a primera vista un sistema capitalista descentralizado), dicho sistema de economía de mercado no es puro, pues la autonomía de los agentes que en él intervienen se encuentra limitada, en mayor o menor medida, por el sistema jurídico que delinea, dirige, supervisa, monitorea y controla su accionar mediante leyes, regulaciones, políticas económicas, controles gubernamentales, etc. (p. 24).

No menos importante es indicar que la Corte Constitucional en la Sentencia C-150 de 2003 ha indicado que la intervención del Estado en el mercado puede ser de diferentes modalidades sin que siempre pueda efectuarse una distinción clara entre las formas de intervención correspondientes. Miremos algunos ejemplos. Global: versa 
sobre la economía como un todo. Sectorial: recae en una determinada área de actividad. Particular: si apunta a una cierta situación como por ejemplo a la de una empresa. Directa: recae sobre la existencia o la actividad de los agentes económicos. Indirecta: cuando está orientada no a la actividad económica propiamente dicha sino al resultado de la misma. Unilateral: cuando el Estado autoriza, prohíbe o reglamenta una actividad económica. Convencional: cuando el Estado pacta con los agentes económicos las políticas o programas que propenden por el interés general. Por vía directiva: cuando el Estado adopta medidas que orientan a los agentes económicos privados. Por vía de gestión: cuando el Estado se hace cargo el mismo de actividades económicas por medio de personas jurídicas generalmente públicas (Sentencia C-150, 2003).

De ahí que como la dirección e intervención del Estado en la economía está presente en todas partes el derecho económico — como aquel derecho de la dirección e intervención estatal en los procesos económicos- también es como el aire por cuanto está en todos lados. Tal explicación a simple vista es comprensible, desde luego se admite cualquier crítica, pues de eso se trata. En este sentido, se finaliza este apartado con señalar que los abogados administrativistas poco a poco están reconociendo que la institución de dirección e intervención del Estado en la economía es temática propia del derecho económico y no del derecho administrativo, entendiendo por este último como el derecho de la administración pública. Vidal Perdomo (1966), por ejemplo, al respecto advirtió lo siguiente:

Una nueva rama del derecho está en formación: el derecho económico. La teoría de la intervención del Estado en la economía, los campos de regulación de la actividad económica de los particulares, la descripción de los engranajes del sector administrativo que tiene responsabilidades en esta área (Banco de la República y otros, por ejemplo), hacen parte de esta disciplina. (p. 4).

\section{Conclusiones}

- El estudio de la discutida relación economía y derecho ya se encuentra superado. Dicha cuestión ya pasó de moda por cuanto ya es evidente y creciente frente a la influencia de elementos económicos en todas las ramas del derecho. Por tanto, hay que darle paso al derecho económico. Debemos ahora desaprender muchas cosas de dicha relación y aprender otras nuevas de este derecho.

- Respecto de si el derecho depende o no de la economía o, si este último (economía) depende o no del primero (derecho), se reconoce que se trata de un tema de discusión intemporal. Pero resulta conveniente estudiar en conjunto entre estas dos áreas de conocimiento en aras de aportar soluciones económicas y jurídicas frente a los problemas sociales. 
- Por eso el estudio y el empleo de las herramientas hermenéuticas económicas y jurídicas resultan trascendentales para el profesional, así como para quienes están en formación, en la medida en que proporcionan elementos de juicio valiosos para ser buenos interlocutores en los discursos tanto académicos como profesionales; con el fin de convertirse en profesionales capaces de intervenir interdisciplinariamente.

- Se debe reconocer que no hay una noción exacta frente a qué se entiende por derecho económico. Algunos lo comprenden como el derecho mixto con respecto al derecho público y el derecho privado. Otros explican que es el derecho de la ordenación económica. O como el derecho de la economía. En este texto se acoge la explicación del derecho económico como el derecho de la intervención del Estado en la economía.

- Consideramos conveniente utilizar la nomenclatura Derecho económico para el curso Economía y derecho que tiene muy mal puesto su nombre, y con ello contribuye a obtener una visión global sobre el derecho. De ahí que se recomienda que en todos los cursos del programa de Derecho analicen las funciones económicas de cada una de las instituciones jurídicas con el propósito de obtener una visión más amplia sobre ellas. Lo anterior se presenta como uno de los aportes del estudio de la epistemología del derecho económico en la formación de abogados.

\section{Referencias}

Alfonso Vargas, L. A. (2020). Seminario doctoral "Argumentación jurídica, teoría y filosofía del derecho". Tunja, Colombia: Universidad Santo Tomás.

Amador Cabra, L. E. (2014). Análisis Económico del Derecho: un paradigma en construcción. En Serie de derecho económico, 1(3), 13-50. Bogotá, Colombia: Ediciones Universidad Externado de Colombia.

Ariño Ortíz, G. (2013). Principios de derecho público económico. Bogotá, Colombia: Ediciones Universidad Externado de Colombia.

Barón Barrera, G. Á. (2016). Transformación del derecho administrativo en derecho económico. Bogotá, Colombia: Ediciones Librería de la U.

Chang, H. (2014). Economía para el 99 \% de la población. Barcelona, España: Ediciones Debate.

Constitución Política de Colombia. (1991). Bogotá, Colombia: Ediciones Legis.

Forero Hernández, C. (2019). Derecho tributario, ¿ derecho administrativo o derecho económico? Dos mil tres mil, 21(1), 7-33. https://doi.org/10.35707/dostresmil/2101

Forero Hernández, C., \& Arana Guaraca, P. (2020). ¿La filosofía política se encuentra en crisis de extinción? Indagare, 1(8), 203-211. https://doi.org/10.35707/indagare/818

Franco Vargas, M. H. (2013). Derecho y economía. Una aproximación teórica. Bogotá, Colombia: Ediciones Leyer. 
Gómez Gallardo, P. (2006). Filosofía del derecho. México: Ediciones IURE Editores.

Gutiérrez Prieto, H. (2001). El análisis económico del derecho. Perspectivas para el desarrollo del derecho económico. En Revista de Derecho, 1(15), 1-61.

Hernández Quintero, H. A. (1991). Delitos económicos en la actividad financiera. Bogotá, Colombia: Ediciones Escuela Rodrigo Lara Bonilla.

Hugo Guerriero, Á. (2007). Filosofía del derecho. Buenos Aires, Argentina: Valletta Ediciones.

Kresalja Roselló, B., \& Ochoa Cardich, C. (2017). Derecho constitucional económico. Lima, Perú: Ediciones Fondo Editorial Pontificia Universidad Católica de Perú PUCP.

Palacios Mejía, H. (1999). La economía en el derecho constitucional colombiano. Bogotá, Colombia: Ediciones Biblioteca Vigente.

Sentencia C-150. (2003). Corte Constitucional de Colombia. Magistrado Ponente: Manuel José Cepeda Espinosa.

Sentencia C-263. (2011). Corte Constitucional de Colombia. Magistrado Ponente: Jorge Ignacio Pretelt Chaljub.

Varó, V. (2013). ¿Para qué sirven realmente los mercados financieros? Madrid, España: Ediciones Paidós. Vidal Perdomo, J. (1966). Derecho administrativo general. Bogotá, Colombia: Ediciones Temis. 